\title{
Kalibrierung von Röntgenbildverstärkern in der computerassistierten orthopädischen Chirurgie
}

\author{
Schauer D., Nolte L.-P.", Kraft M., Boenick U. \\ Institut für Mikrotechnik und Medizintechnik, Technische Universität Berlin \\ "Maurice E.Müller Institut für Biomechanik, Universităt Bern
}

\section{EINLEITUNG}

Die computerassistierte orthopädische Chirurgie (CAOS) nutzt eine mathematische Simulation der Projektion von Instrumenten und anatomischen Strukturen in prä- oder intraoperativ erstellte Röntgenoder CT-Bilder. Der Navigation liegt eine Signalübertragung durch mindestens vier Infrarotlichtstrahler (LED) pro Systemkomponente zugrunde, welche in einem Trägerschild fixiert ein lokales Koordinatensystem aufspannen. In diesem ist die Komponente als Starkörper definiert. Die Signale werden von einem optoelektronischen Positionssensor (OPTOTRAK 3020, Northern Digital) erfaßt und durch ein Datenverarbeitungssystem aufbereitet [1].

Grundlage einer effizienten Implementierung der CAOS in die traumatologische orthopädische Chirurgie ist die Nutzung von Fluoroskopen zur Bilderfassung. In Abb. 1 sind die erforderlichen Transformationen zwischen den Systemkomponenten dargestellt.

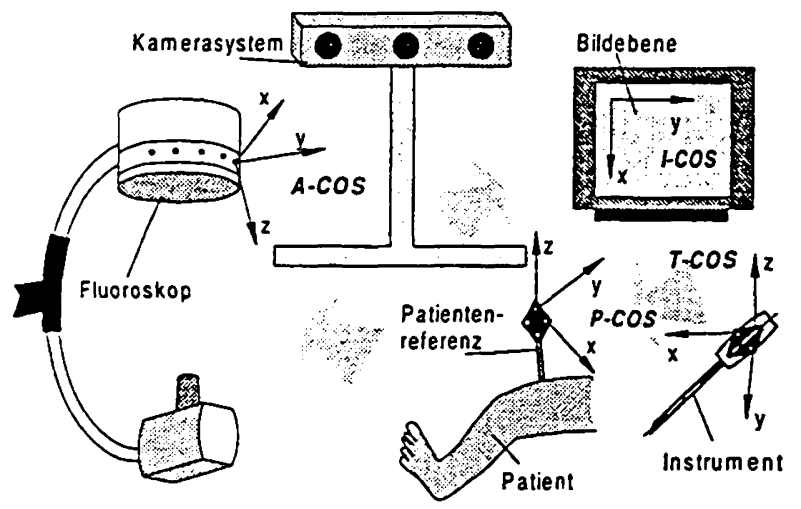

Abb. I Transformationen der Koordinatensysteme

\section{KALIBRIERUNG}

Durch Fluoroskope aufgenommene Röntgenbilder sind durch die geometrisch-technischen Gegebenheiten des C-Arms sowie den Aufbau des Bildverstärkers verzerrt (extrinsische sowie intrinsische Verzerrungen). Es ist möglich, diese Verzerrungen durch präoperative Kalibrierung in einer definierten Ausgangslage zu kompensieren und so eine lineare Projektion der Relativbewegungen in die Bildebene zu ermöglichen.

Zur extrinsischen Kalibrierung werden zwei Aufnahmen einer Markerplatte mit ihren äquivalenten räumlichen Lagen mathematisch verknüpft, so $\mathrm{da} B$ der virtuelle Fokuspunkt der Strahlungsquelle bestimmbar ist. Die intrinsische Kalibrierung erfolgt durch Interpolation der Markerprojektionen einer Kalibrierplatte in möglichst geringem Abstand vom Eingangsphosphor des Bildverstärkers. Bezüglich der Ausgangsgeometrie der Kalibrierplatten können Algorithmen zur Rücksubstitution der Verzerrungen aufgestellt und so weitere Röntgenaufnahmen entzern werden.

Bedeutenden EinfluB auf die Verzerrung von intraoperativ erstellten Röntgenbildern haben die mechanische Deformation des C-Arms sowie externe Magnetfelder (Erdmagnetfeld, elektro-magnetische Felder).

\section{ZIELSTELLUNG}

Es war ein geschlossenes Konzept zur Kalibrierung von Röntgenbildverstärkern aufzustellen, welches folgende Systemkomponenten umfaßt:

- (optimierte) extrinsiche Kalibrierung,

- (optimierte) intrinsichen Kalibrierung,

- Kalibrierung der mechanischen Deformation,

- Magnetfeld-Kalibrierung.

Der Hauptschwerpunkt der Konzepterstellung lag in der Erfassung und Kalibrierung der mechanischen Deformation.

\section{LÖSUNGSANSATZ}

Nach einer Diskussion verschiedener Verfahren der Erfassung räumlicher Bewegungen (optisch, elektronisch und/oder mechanisch), einer Abschätzung der Meßbereiche sowie der erforderlichen Genauigkeiten, wurden folgende Lösungsvarianten zur Erfassung der mechanischen Deformation des C-Arms entwickelt und näher analysiert:

\section{a) Mechanische Messung mittels Meßtastern}

Ein an der Strahlungsquelle fixierter Meßstab wird in einem am Bildverstärker befestigten und in dessen Koordinatensystem $A-C O S$ definierten MeBzylinder von elektronischen MeBtastern in verschiedenen Ebenen angelenkt. Ein Berechnungsalgorithmus ermöglicht die Rücksubstitution der mechanischen Deformation äquivalent zur Auslenkung der Taster. Der maximale sowie mittlere Fehler der Messung ist abhängig von der Meßgenauigkeit der Taster, der Größenordnung der mechanischen Deformation sowie der Fertigungsgenauigkeit der Einzelkomponenten. Basierend auf der extrinsischen Kalibrierung ist die Fokuspunktverschiebung durch Bestimmung der Starrkörperbewegung berechenbar. 
b) Optoelektronische Positionsdetektion (Optotrak)

Durch einmalige extrinsischer Kalibrierung des Bildverstärkers ist der räumliche Vektor des Fokuspunktes ( $p_{\text {Fokur. } A}$ ) im Koordinatensystem A-COS bestimmbar. An der Strahlungsquelle ist ein LED-Schild fixiert, welches das Koordinatensystem S-COS bildet und die Bestimmung des Ortsvektors des Fokuspunktes ( $\left.p_{\text {Fonku.s. }}\right)$ in diesem Koordinatensystem durch Koordinatentransformation ermöglicht. Die Rotationsmatrix (R) sowie der Translationsvektor ( $t$ ) der Transformation werden in Real-Zeit durch den Positionssensor aufgestellt.

$$
\mathbf{p}_{\text {Frous. } S}=\mathbf{R} \cdot \mathbf{p}_{\text {Fotus. } A}+\mathbf{t}
$$

Dieser Vektor ist konstant (Starrkörperhypothese) und kann bei Lageänderung des C-Arms durch Rücktransformation in das Koordinatensystem des Bildverstärkers (p' Foku; $A$ ) zur Berechnung des Verschiebungsvektors (s) genutzt werden.

$$
\begin{aligned}
& \mathbf{p}_{\text {Fokus.A }}^{\prime}=\mathbf{R}^{\prime} \cdot\left(\mathbf{p}_{\text {Folus. } s}-\mathbf{t}^{\prime}\right) \\
& \mathbf{S}=\mathbf{p}_{\text {Fokus. } A}-\mathbf{p}_{\text {Folkus. } A}
\end{aligned}
$$

Durch Optimierung der in Abb. 2 dargestellten Starrkörperparameter ist der Fehler der Koordinatentransformation zu minimieren.

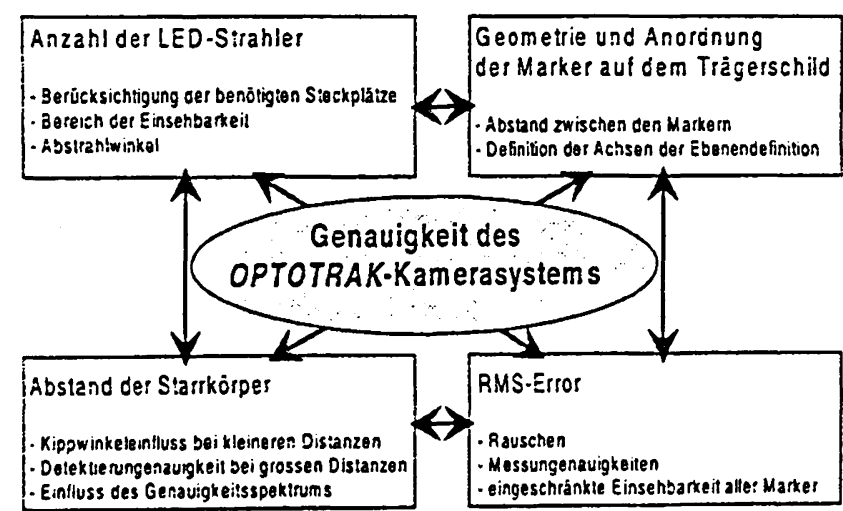

Abb. 2 Parameter der Starrkörpertransformation

\section{c) Interpolation extrinsischer Kalibrierungen}

Durch Entwicklung einer optimierten Kalibriereinheit und Minimierung der erforderlichen Transformationsschritte konnten der statische Fehler (Abweichung einer wiederholten extrinsische Kalibrierung nach einmaliger Raumdatenerfassung der Kalibrierebenen) sowie der dynamische Fehler (wiederholte Raumdatenerfassung der Kalibrierebenen mit nachfolgender einmaliger extrinsischer Kalibrierung) minimiert werden (Abb. 3).

Durch wiederholte Fokuspunktbestimmung in verschiedenen Lagen des C-Arms und anschließender Interpolation zwischen diesen Stützpunkten ist die mechanische Deformation in Abhängigkeit von der räumlichen Lage des C-Arms zu ermitteln.

Die Anwendbarkeit des Verfahrens hängt im besonderen Maße von der Genauigkeit der Raumdatenerfassung der Kalibrierebenen sowie der Anzahl der Stüzzunkte, welche der Interpolation zugrunde liegen, ab.

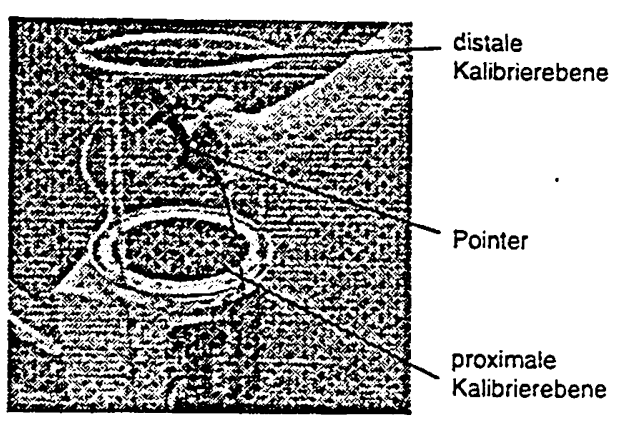

Abb. 3 Modifizierte extrinsische Kalibrierung

Es ergaben sich folgende maximale sowie mittlere Fehler einer Bestimmung der mechanischen Deformation:

\begin{tabular}{|c|c|c|}
\hline Verfahren & max. Fehler & mittlerer \\
\hline a) & & Fehler \\
\hline b) & $2.46 \mathrm{~mm}$ & $\approx 1.300 \mathrm{~mm}$ \\
\hline c) & $0.72 \mathrm{~mm}$ & $0.417 \mathrm{~mm}$ \\
\hline
\end{tabular}

\section{KONZEPT DER KALIBRIERUNG}

Die extrinsische Kalibrierung wird mittels der in Abb. 3 dargestellten Kalibriereinheit und des äquivalent hierzu entwickelten Zeigers zur Raumdatenerfassung der Kalibrierebenen durchgeführt. Die intrinsische Kalibrierung erfolgt in jeder Aufnahme durch eine kubische Spline-Interpolation von Markerprojektionen einer permanent auf dem Bildverstärker fixierten Kalibrierplatte, welche nach Korrektur aus der Bildebene substituiert werden. Diese Vorgehensweise ermöglicht im weiteren eine flexible MagnetfeldKalibricrung (Magnetfeldeinflüsse sind quantitativ nicht bestimmbar). Die mechanische Deformation kann durch die Verfahen:

- optoelektronische Positionsdetektion (b) sowie

- Interpolation extrinsischer Kalibrierungen (c)

erfolgen. Welches beider Verfahren genutzt wird hängt von der verfügbaren Stützpunktzahl, dem Interpolationsverfahren sowie der Kameragenauigkeit ab.

\section{ZUSAMMENFASSUNG}

Durch die Entwicklung der computerassistierten orthopädischen Chirurgie wurde ein den Patienten sowie das Operationspersonal vor hohen Strahlungsbelastungen schützendes System entwickelt. welches eine maximale Arbeitsgenauigkeit des Chirurgen gewährleistet. Durch die Anwendung von Röntgenbildverstärkern zur Bilderstellung ist eine flexible Nutzung des Systems in der orthopädischen sowie Unfallchirurgie möglich.

\section{LITERATUR}

[1] R. Hofstetter, M. Slomczykoushi L.-P. Nolte

Fluoroskopy Based Surgical Navigation

in: Computer Assisted Radiology and Surgery (CAR'y7) 